\title{
Coarse-Grained Simulations of Flow-Induced Nucleation in Semicrystalline Polymers
}

\author{
Richard S. Graham ${ }^{1}$ and Peter D. Olmsted ${ }^{2}$ \\ ${ }^{1}$ School of Mathematical Sciences, University of Nottingham, Nottingham NG7 2RD, United Kingdom \\ ${ }^{2}$ School of Physics and Astronomy, University of Leeds, Leeds LS2 9JT, United Kingdom \\ (Received 30 March 2009; revised manuscript received 15 July 2009; published 10 September 2009)
}

\begin{abstract}
We perform kinetic Monte Carlo simulations of flow-induced nucleation in polymer melts with an algorithm that is tractable even at low undercooling. The configuration of the noncrystallized chains under flow is computed with a recent nonlinear tube model. Our simulations predict both enhanced nucleation and the growth of shish-like elongated nuclei for sufficiently fast flows. The simulations predict several experimental phenomena and theoretically justify a previously empirical result for the flow-enhanced nucleation rate. The simulations are highly pertinent to both the fundamental understanding and process modeling of flow-induced crystallization in polymer melts.
\end{abstract}

DOI: 10.1103/PhysRevLett.103.115702

PACS numbers: 64.70.km, 64.60.qe, 83.80.Sg

Introduction.-The nucleation of microscopic crystallites in polymer liquids is profoundly influenced by flow $[1,2]$. This flow-induced crystallization (FIC), is a fascinating example of an externally driven, nonequilibrium phase transition, controlled by kinetics. Furthermore, FIC is ubiquitous in industrial processing of semicrystalline polymers, the largest group of commercially useful polymers. A fundamental understanding of FIC promises extensive control of polymer solid state properties, as virtually every property of practical interest is determined by the crystal morphology. Flow can drastically enhance nucleation and trigger the formation of highly aligned, elongated crystals, known as shish kebabs [1]. Recent experiments on entangled polymers have studied, in detail, shish kebab formation [3-5] and the role of blend concentration [6], molecular architecture [7], and molecular relaxation time [8]. Often the most pronounced flow-induced effects occur near the melting point, where quiescent crystallization is immeasurably slow $[2,8]$.

The widely postulated mechanism for FIC states that flow forces the polymer chains into elongated configurations, which lowers the entropic penalty for crystallization [1]. However, this hypothesis has yet to be developed into a quantitative molecular model. FIC is extremely sensitive to the flow-induced configurations of the noncrystalline chains, so an accurate molecular flow model is an essential prerequisite. Unfortunately, most polymer flow models predict only the macroscopic stress tensor and not the full molecular configuration. Alternatively, detailed simulations of polymer crystallization have provided much useful information on the growth process [9-11], yet simulating primary nucleation has proven difficult, especially at low undercooling, because of the extremely long nucleation times. At a much higher level of coarse graining, models based on differential equations either assume an empirical dependence of the nucleation rate on the flow conditions [12], the stress tensor [13], or the chain stretch [14] or assert that free energy changes under flow can be directly subtracted from the nucleation barrier $[15,16]$. In either case the postulated FIC mechanism remains untested. An intermediate level of coarse graining is required to surmount these difficulties.

This Letter presents coarse-grained kinetic Monte Carlo (MC) [17] simulations of anisotropic nucleation in flowing polymers. We compute chain configurations using a recent molecular flow model [18] that reliably predicts both neutron scattering $[19,20]$ and bulk stresses. Our simulations predict both enhanced nucleation and elongated shish nuclei. Kinetic MC has previously been used to model quiescent crystal growth in dilute polymers [21]. However, it is particularly suited to nucleation, and our algorithm is tractable even at low undercooling, providing an efficient and highly flexible framework to simulate general anisotropic nucleation under external fields.

Model.-We compute the transient chain configuration of the uncrystallized chains under flow using the Graham, Likhtman and Milner, McLeish (GLaMM) model [18], with finite chain extensibility included using Cohen's approximation [22]. The chains are divided into $Z$ subchains, each corresponding to an entanglement segment of $N_{e}$ Kuhn steps of length $b$. We take $N_{e}=100$ throughout this Letter. One deterministic run of the model provides the end-to-end vector $\mathbf{f}_{i}(t)=\left\langle\mathbf{r}_{i} \mathbf{r}_{i}\right\rangle$, where the ensemble average is for subchains of type $i$. The data for an entire transient flow are used later in the nucleation simulations. All flow time scales are in units of the subchain Rouse time $\tau_{e}$, and $\mathbf{r}$ is normalized by $\sqrt{N_{e}} b$.

Deformation of the amorphous chains has two effects on the nucleation kinetics: stretching reduces the entropic penalty for crystallization; and monomer alignment modifies the probability of compatible alignment with the nucleus. The change in elastic free energy $\Delta F^{\mathrm{el}}$ for chains with ensemble average constraints $\mathbf{f}=\langle\mathbf{r r}\rangle$, but locally at equilibrium, can be calculated by statistical mechanics [23]. Although an analytic calculation is not possible for finitely extensible chains, steep free energy gradients in 
highly stretched chains suppress fluctuations. Thus our numerical calculations for uniaxial deformations show that $\Delta F^{\mathrm{el}}(\langle\mathbf{r r}\rangle)$ can be accurately approximated by an expression that interpolates between Gaussian elasticity [23] for small Trf and Cohen's [22] approximation with $\mathbf{r}^{2}=\operatorname{Tr} \mathbf{f}$ at high stretching,

$$
\Delta F^{\mathrm{el}}=\frac{1}{2} \operatorname{Tr} \mathbf{f}-\frac{1}{2} \operatorname{Tr} \ln \mathbf{f}-N_{e} \ln \left(1-\frac{\operatorname{Tr} \mathbf{f}}{N_{e}}\right) .
$$

Similarly, numerical calculation of the monomer orientation distribution $w(\theta)$ for chains with a constraint $\mathbf{f}$ are well approximated by using $\mathbf{r}^{2}=\operatorname{Tr} \mathbf{f}$ in the expression for $w(\theta)$ derived from a direct constraint on $\mathbf{r}$ [24].

$$
w(\theta)=\frac{\mathcal{L}^{-1}\left[\sqrt{\operatorname{Trf}} / N_{e}\right]}{4 \pi \sinh \left(\mathcal{L}^{-1}\left[\sqrt{\operatorname{Trf}} / N_{e}\right]\right)} \cosh \left(\mathcal{L}^{-1}\left[\frac{\sqrt{\operatorname{Trf}}}{N_{e}}\right] \cos \theta\right),
$$

where $\mathcal{L}^{-1}$ is the inverse Langevin function and $\theta$ is the angle between the monomer and the principle axis of $\mathbf{f}$. Each subchain has an individual $\mathbf{f}_{i}$ and so is treated as a separate species with concentration $\phi_{i}=1 / Z$.

Our coarse-grained simulations use the minimal nucleus description required for anisotropic nucleation. The nucleus comprises $N_{T}$ crystallized "monomers" or Kuhn steps, arranged in stems, with each stem formed from a single chain (see Fig. 1). The total number of stems $N_{s}$ and the number of monomers in each stem are simulated. The arrangement of the monomers within the crystal is not resolved. The nucleus is assumed to be spheroidal with the polar radius $L$ parallel to the stems. Assigning a crystalline volume of $b_{0}^{3}$ to each monomer and normalizing all lengths by $b_{0}$ give the equatorial radius $W=\sqrt{N_{s} / \pi}$, the volume $V=N_{T}$, and thus the polar radius $L=\frac{3 N_{T}}{4 N_{s}}$. We also simulate the unit vector $\hat{\mathbf{v}}$ parallel to the polar radius. As in classical nucleation theory the nucleus free energy comprises the free energy of crystallization, proportional to the nucleus volume, and a free energy penalty proportional to the surface area $S$. Thus the free energy in units of $k_{B} T$ is $\mathcal{F}\left(N_{T}, N_{s}\right)=-\epsilon_{B} N_{T}+\mu_{S} S$, where $\epsilon_{B}$ and $\mu_{s}$ are the coefficients of the volume and surface area free energies, respectively.

We simulate two types of MC moves: the addition of a new stem containing one monomer and the lengthening of an existing stem by adding a new monomer, each with a corresponding reverse move. As in [24] we assume that to

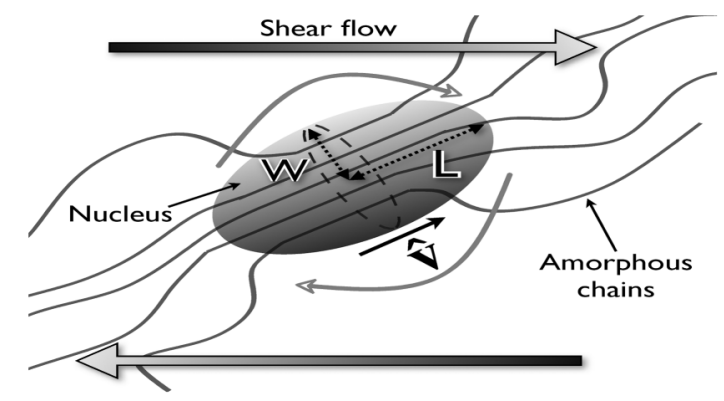

FIG. 1. A sheared nucleus with protruding amorphous chains. attach, a monomer must be oriented within a solid tolerance angle $\Omega$ of the nucleus orientation. For small $\Omega$, the fraction of monomers within this angle is $w(\theta) \Omega$, where $\theta$ is the angle between the nucleus polar radius and the subchain principle strain axis. The stem attachment rate for species $i$ is proportional to its melt concentration $\phi_{i}$. In contrast, for stem lengthening only the next monomer along the chain forming the stem can crystallize. The concentration of this monomer at the nucleus surface where the lengthening event occurs is taken to be unity. Thus the attachment $(+)$ and detachment $(-)$ move rates for stem addition $\left(k_{\mathrm{st}}\right)$ and stem lengthening $\left(k_{\text {len }}\right)$ are

$$
\begin{aligned}
k_{\mathrm{st}}^{+} & =\frac{1}{\tau_{0}} \phi_{i} \omega_{i} \min \left(1, e^{-\Delta \mathcal{F}_{\mathrm{st}}^{+}}\right) \quad k_{\mathrm{st}}^{-}=\frac{1}{\tau_{0}} \min \left(1, e^{-\Delta \mathcal{F}_{\mathrm{st}}^{-}}\right) \\
k_{\text {len }}^{+} & =\frac{1}{\tau_{0}} \omega_{i} \min \left(1, e^{-\Delta \mathcal{F}_{\text {len }}^{+}}\right) \quad k_{\text {len }}^{-}=\frac{1}{\tau_{0}} \min \left(1, e^{-\Delta \mathcal{F}_{\text {len }}^{-}}\right),
\end{aligned}
$$

where $\tau_{0}$ is the time for a monomer attachment attempt, $\omega_{i}=4 \pi w_{i}\left(\theta_{i}\right)$ is the fraction of correctly aligned monomers (normalized to unity in the quiescent limit), and the constant $\ln (\Omega / 4 \pi)$ has been added to $\epsilon_{B}$. The free energy change of attaching a new stem of species $i$ is

$$
\Delta \mathcal{F}_{\text {st }}^{+}=\mathcal{F}\left(N_{T}+1, N_{s}+1\right)-\mathcal{F}\left(N_{T}, N_{s}\right)-\frac{1}{N_{e}} \Delta F_{i}^{\mathrm{el}}\left(\mathbf{f}_{i}\right),
$$

where $\Delta F_{i}^{\mathrm{el}}$ is the flow-induced free energy change in subchain $i$. Similar calculations give the free energy changes for the other move types. A stem can only be detached if it contains a single monomer.

The kinetic MC algorithm requires a sum over all possible move rates. The area available for stem addition moves is $\mathcal{A}\left(N_{s}\right)$, which is taken to be proportional to $\sqrt{N_{s}}$, and to give spherical nuclei in the quiescent limit. To obey detailed balance the rate of stem removal must be multiplied by $\mathcal{A}\left(N_{s}-1\right) / N_{s}$, the probability of a given stem being at the nucleus surface. Each stem can lengthen or shorten from either the top or bottom. Thus the total sum over all possible move rates is

$$
\begin{aligned}
K_{\text {Total }}= & \mathcal{A}\left(N_{s}\right) \sum_{i=1}^{Z}\left(k_{\mathrm{st}}^{+}\right)_{i}+\frac{\mathcal{A}\left(N_{s}-1\right)}{N_{s}} \sum_{j=1}^{N_{s}}\left(k_{\mathrm{st}}^{-}\right)_{j} \\
& +\sum_{j=1}^{N_{s}}\left[\left(k_{\text {len }}^{+ \text {top }}\right)_{j}+\left(k_{\text {len }}^{- \text {top }}\right)_{j}+\left(k_{\text {len }}^{+ \text {bot }}\right)_{j}+\left(k_{\text {len }}^{- \text {bot }}\right)_{j}\right] .
\end{aligned}
$$

At each kinetic MC time step one move is performed at random, with the selection probability weighted by the move rate. Time is then incremented by a stochastically determined interval $\Delta t=-\ln \zeta / K_{\text {Total }}$, where $\zeta$ is chosen uniformly on $[0,1][17,21]$.

After each MC step the nucleus orientation $\hat{\mathbf{v}}$ is incremented over $\Delta t$ by Brownian dynamics. Flow rotates the spheroid nucleus through the Jeffery algorithm [25]. The angular diffusion time $\tau_{\text {rot }}$ scales with both the nucleus volume $N_{T}$ and aspect ratio $\rho$ following the expressions in [25], giving $\tau_{\text {rot }}=\alpha \tau_{0} N_{T} G(\rho)$, where $G(\rho)$ depends only on the aspect ratio and we have introduced a dimensionless 
constant $\alpha$, connecting $\tau_{\text {rot }}$ with the monomer attachment time $\tau_{0}$. As the Jeffery algorithm is for Newtonian fluids, we have neglected non-Newtonian effects here. After each time step all $\mathbf{f}_{i}$ values are updated from the GLaMM model results, and $\Delta F_{i}^{\mathrm{el}}$ and $\omega_{i}$ are recalculated. The parameter $\mathcal{S}=\tau_{e} / \tau_{0}$ sets the ratio of flow and monomer attachment time scales. We use $\mathcal{S}=10$ throughout this Letter, unless indicated otherwise, although similar results are obtained for any value of $\mathcal{S}>1$.

Results.-Each simulation evolves a single nucleus from a single monomer. The algorithm is especially effective at low undercooling; as for small nuclei the rate sum is small, leading to large time steps. The quiescent free energy landscape can be calculated analytically from $\epsilon_{B}$ and $\mu_{S}$ to give a dimensionless nucleation barrier $\Delta f^{*}$ and a critical nucleus of $n^{*}$ monomers. The simulated nucleation time $\tau_{N}$ is the first time the polar and equatorial radii simultaneously exceed the critical radius $r^{*}=\sqrt[3]{3 n^{*} / 4 \pi}$. Choosing a larger threshold size for nucleation has little effect on $\left\langle\tau_{N}\right\rangle$. The results are accurately approximated by $\left\langle\tau_{N}\right\rangle=\tau_{0} \exp \left(\Delta f^{*}\right)$. We obtained good statistics for barriers up to $25 k_{B} T$ in $\sim 50 \mathrm{hrs}$ on one $2 \mathrm{GHz}$ processor, giving a nucleation time of $\sim 10^{11} \tau_{0}$. The nucleation times are Poisson distributed, so the nucleation rate can be defined as $\dot{N}_{0}=1 /\left\langle\tau_{N}\right\rangle$.

Under flow, the nucleation kinetics depend on the evolving chain deformation. We define the instantaneous nucleation rate $\dot{N}(t) \approx \frac{1}{1-n(t)} \frac{n(t+\Delta t)-n(t-\Delta t)}{2 \Delta t}$ where $n(t)$ is the cumulative fraction of runs nucleated at time $t$. For shear rates $\dot{\gamma}$ that are slow compared to the critical nucleus rotation time $\left(\tau_{\text {rot }}^{n^{*}} \dot{\gamma} \ll 1\right)$, alignment effects can be ignored, equivalent to taking $\alpha=0$. The GLaMM model predictions for startup of constant shear at $\dot{\gamma} \tau_{e}=0.1$ of a $Z=25$ melt and the resulting instantaneous nucleation rate are in Fig. 2(a). The results are independent of the ratio of flow and crystallization time scales for $\mathcal{S} \geqslant 1$. In fact, fixing the noncrystalline chain configuration to that corresponding to flow time $t$ for the entire simulation and plotting the resulting quasistatic nucleation rate against $t$ reproduces the transient results [Fig. 2(a)]. Thus nucleation is fully controlled by the instantaneous configuration of the surrounding chains, if $\tau_{e}>\tau_{0}$.

The stretch ratio $\lambda=\frac{1}{Z} \sum_{i=1}^{Z} \sqrt{\left\langle\mathbf{r}_{i}^{2}\right\rangle}$ is the dominant factor determining the nucleation rate, despite variations in flow rate, molecular weight, and flow geometry [Fig. 2(b)]. This universal result is somewhat surprising as the distribution of stretch along the chain varies considerably with flow conditions, which may be expected to influence nucleation, especially if molecular weight and flow geometry are varied. This result will be useful in deriving simple differential models of FIC [13,14] as the nucleation rate can be described by the expression $\dot{N}=\dot{N}_{0} \exp \left[\eta\left(\lambda^{2}-\right.\right.$ 1)], where $\eta$ is a fitting constant. Also in Fig. 2(b) is the curve $\dot{N}=\dot{N}_{0}+\beta\left(\lambda^{4}-1\right)$, which empirically fits measured nucleation rates from flowing melts [14]. The agreement with our simulation data for $\lambda \lesssim 3.5$ theoretically justifies this empirical expression. In the inset to Fig. 2(b) the sensitivity of nucleation to chain stretching increases with decreased undercooling (decreasing $\epsilon_{B}$ ), as seen experimentally [26]. Figure 2(c) directly compares our simulations with steady-state nucleation rate measurements on a polydisperse isotactic polypropylene sample during shear [27]. The parameter determination, projection of the simulation to large energy barriers, and approximation of the molecular weight distribution as a bimodal blend are detailed in the supplementary material [28]. The close agreement shows our model can quantitatively account for FIC measurements. Figure 2(a) also shows the transient nucleation rate when $\alpha=5$. This higher $\alpha$ value gives slower angular diffusion, meaning that flow aligns subcritical nuclei, accelerating nucleation. Here, the quasistatic nucleation remains Poissonian and matches the transient rates, although $\dot{N}$ now depends on both $\lambda$ and $\dot{\gamma} \tau_{\text {rot }}^{n^{*}}$. Further increases of $\alpha$ give almost identical results.

In experiments shish nuclei are especially prevalent in melts of short chains blended with a small amount of very long chains [4-8]. We simulated a melt of $Z=15$ chains blended with $2 \mathrm{wt} \%$ of $Z=52$ chains, using a generalization of the GLaMM model to bimodal blends [29]. High shear produces very elongated nuclei (Fig. 3) from a purely
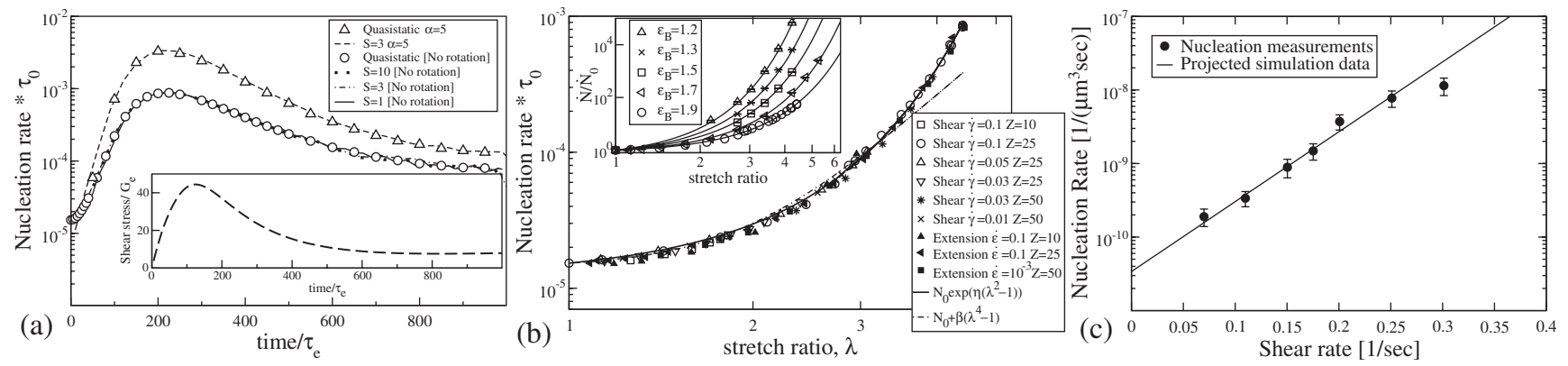

FIG. 2. (a) Transient and quasistatic nucleation rates for a $Z=25$ monodisperse melt with $\epsilon_{B}=1.9$ and $\mu_{S}=1.9$ under startup shear at $\dot{\gamma} \tau_{e}=0.1$; inset contains GLaMM model predictions for shear stress ( $G_{e}$ is the shear modulus). (b) Master curve of nucleation rate against stretch ratio ( $\dot{\epsilon}$ is the extension rate); inset shows the master curves for varying $\epsilon_{B}$. (c) Steady-state nucleation rate measurements against shear rate for an industrial polydisperse isotactic polypropylene melt [27] compared with projected simulation results. 


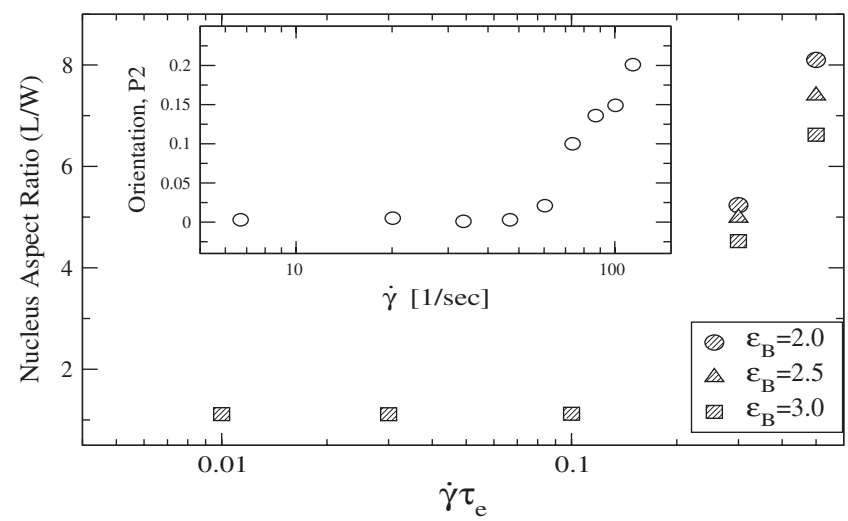

FIG. 3. Simulated nucleus aspect ratio at point of nucleation against shear rate for a $2 \%$ high-molecular-weight blend ( $\alpha=$ 5.0, $\mu_{S}=2.5$, sheared for $120 \tau_{e}$ ); inset shows experimental orientation data on a bimodal blend sheared for $20 \mathrm{sec}$ [8].

kinetic mechanism. The shish widen by adding new stems using any monomer from the melt, whereas shish lengthen by adding monomers along an existing stem. Therefore the concentration of monomers from stretched segments at the growth surface is greater for lengthening than for widening, provided the nucleus contains a disproportionate number of stretched segments. Fast flow conditions are required for this disparity to overcome the significant surface area cost of elongated nuclei. Figure 3 (inset) shows experimental data for an orientational order parameter, the P2 orientation function, against shear rate for a $2 \%$ highmolecular-weight blend [8], highlighting similarities with our predictions. Also in Fig. 3 reduced undercooling, from reducing $\epsilon_{B}$, increases the anisotropy, as seen experimentally [30]. When crystallization is less favorable, the disparity in the kinetics of stretched and unstretched chain segments increases.

Discussion.-Our efficient kinetic Monte Carlo algorithm for flow-induced nucleation in polymer melts is tractable even at low undercooling. The flow-induced nucleation rate is a universal function of the chain stretch ratio, independent of flow rate, molecular weight, and flow geometry, but with decreasing undercooling causing increased flow sensitivity. This universal curve is very similar to an empirical relationship that accurately describes flow-induced nucleation rate measurements [14], justifying this empirical result. Our successful quantitative comparison with nucleation measurements [27] establishes that changes in chain free energy under flow can describe flow-enhanced nucleation in semicrystalline polymers. In our bimodal blend simulations, a few percent of highmolecular-weight chains optimize shish formation, and the degree of anisotropy increases with shear rate and decreased undercooling, all of which are seen experimentally $[4-8,30]$. The simulation can readily be generalized to fully polydisperse melts, an essential step to model industrial polymer processing. The efficiency and flexibility of our algorithm make it suitable to simulate general anisotropic nucleation under external fields.

We thank the EPSRC for funding (GR/T11807/01) and L. Balzano, R. Steenbakkers, G. Peters, O. Mykhaylyk, T. Ryan, and T. McLeish for useful discussions.

[1] A. Keller and H. W. Kolnaar, in Processing of Polymers, edited by H. Meijer (Wiley, Weinheim, 1997).

[2] F. L. Binsbergen, Nature (London) 211, 516 (1966).

[3] S. Kimata et al., Science 316, 1014 (2007).

[4] B. S. Hsiao et al., Phys. Rev. Lett. 94, 117802 (2005).

[5] L. Balzano et al., Phys. Rev. Lett. 100, 048302 (2008).

[6] M. Seki et al., Macromolecules 35, 2583 (2002).

[7] E. L. Heeley et al., Macromolecules 39, 5058 (2006).

[8] O. O. Mykhaylyk et al., Macromolecules 41, 1901 (2008).

[9] N. Waheed, M. J. Ko, and G. C. Rutledge, Polymer 46, 8689 (2005).

[10] W. Hu, D. Frenkel, and V. Mathot, Macromolecules 35, 7172 (2002).

[11] J. Zhang and M. Muthukumar, J. Chem. Phys. 126, 234904 (2007).

[12] G. Eder and H. Janeschitz-Kriegl, in Processing of Polymers, edited by H.E.H. Meijer (Wiley, Weinheim, 1997).

[13] H. Zuidema, G.W.M. Peters, and H.E.H. Meijer, Macromol. Theory Simul. 10, 447 (2001).

[14] R. J. A. Steenbakkers, G. W. M. Peters, and H.E.H. Meijer, in The XV International Congress On Rheology: The Society of Rheology 80th Annual Meeting AIP Conference Proceedings (American Institute of Physics, Monterey, 2008), Vol. 1027, p. 493.

[15] S. Coppola, N. Grizzuti, and P. L. Maffettone, Macromolecules 34, 5030 (2001).

[16] J. Kulkarni and A. Beris, J. Non-Newtonian Fluid Mech. 82, 331 (1999).

[17] D. T. Gillespie, J. Phys. Chem. 81, 2340 (1977).

[18] R. S. Graham et al., J. Rheol. 47, 1171 (2003).

[19] J. Bent et al., Science 301, 1691 (2003).

[20] A. Blanchard et al., Phys. Rev. Lett. 95, 166001 (2005).

[21] J. P. K. Doye and D. Frenkel, Phys. Rev. Lett. 81, 2160 (1998).

[22] A. Cohen, Rheol. Acta 30, 270 (1991).

[23] P. D. Olmsted and S. T. Milner, Macromolecules 27, 6648 (1994).

[24] L. Jarecki, in Progress in Understanding of Polymer Crystallization, edited by G. Reiter and G. R. Strobl (Springer, Berlin, 2007), Vol. 714, p. 65.

[25] L. G. Leal and E. J. Hinch, J. Fluid Mech. 46, 685 (1971).

[26] S. Coppola et al., Polymer 45, 3249 (2004).

[27] I. Coccorullo, R. Pantani, and G. Titomanlio, Macromolecules 41, 9214 (2008).

[28] See EPAPS Document No. E-PRLTAO-103-051939 for details of this comparison with experiments. For more information on EPAPS, see http://www.aip.org/pubservs/ epaps.html.

[29] R. S. Graham et al., Soft Matter 5, 2383 (2009).

[30] L. Balzano, Ph.D. thesis, TU/e (Eindhoven) (2008). 